\title{
Discovery of extended VHE gamma-ray emission from the asymmetric pulsar wind nebula in MSH 15-52 with HESS
}

\author{
F. Aharonian ${ }^{1}$, A. G. Akhperjanian ${ }^{2}$, K.-M. Aye ${ }^{3}$, A. R. Bazer-Bachi ${ }^{4}$, M. Beilicke $^{5}$, W. Benbow ${ }^{1}$, D. Berge ${ }^{1}$,
} P. Berghaus ${ }^{6, \star}$, K. Bernlöhr ${ }^{1,7}$, C. Boisson ${ }^{8}$, O. Bolz ${ }^{1}$, I. Braun ${ }^{1}$, F. Breitling ${ }^{7}$, A. M. Brown ${ }^{3}$, J. Bussons Gordo ${ }^{9}$, P. M. Chadwick ${ }^{3}$, L.-M. Chounet ${ }^{10}$, R. Cornils ${ }^{5}$, L. Costamante ${ }^{1,20}$, B. Degrange ${ }^{10}$, A. Djannati-Atai ${ }^{6}$, L. O'C. Drury ${ }^{11}$, G. Dubus ${ }^{10}$, D. Emmanoulopoulos ${ }^{12}$, P. Espigat ${ }^{6}$, F. Feinstein ${ }^{9}$, P. Fleury ${ }^{10}$, G. Fontaine ${ }^{10}$, Y. Fuchs ${ }^{13}$, S. Funk ${ }^{1}$, Y. A. Gallant ${ }^{9}$, B. Giebels ${ }^{10}$, S. Gillessen ${ }^{1}$, J. F. Glicenstein ${ }^{14}$, P. Goret ${ }^{14}$, C. Hadjichristidis ${ }^{3}$, M. Hauser ${ }^{12}$, G. Heinzelmann ${ }^{5}$, G. Henri ${ }^{13}$, G. Hermann ${ }^{1}$, J. A. Hinton ${ }^{1}$, W. Hofmann ${ }^{1}$, M. Holleran ${ }^{15}$, D. Horns ${ }^{1}$,

O. C. de Jager ${ }^{15}$, B. Khélifi ${ }^{1}$, Nu. Komin ${ }^{7}$, A. Konopelko ${ }^{1,7}$, I. J. Latham ${ }^{3}$, R. Le Gallou ${ }^{3}$, A. Lemière 6 ,

M. Lemoine-Goumard ${ }^{10}$, N. Leroy ${ }^{10}$, T. Lohse ${ }^{7}$, O. Martineau-Huynh ${ }^{16}$, A. Marcowith ${ }^{4}$, C. Masterson ${ }^{1,20}$,

T. J. L. McComb ${ }^{3}$, M. de Naurois ${ }^{16}$, S. J. Nolan ${ }^{3}$, A. Noutsos ${ }^{3}$, K. J. Orford ${ }^{3}$, J. L. Osborne ${ }^{3}$, M. Ouchrif ${ }^{16,20}$, M. Panter ${ }^{1}$, G. Pelletier ${ }^{13}$, S. Pita ${ }^{6}$, G. Pühlhofer ${ }^{1,12}$, M. Punch $^{6}$, B. C. Raubenheimer ${ }^{15}$, M. Raue ${ }^{5}$, J. Raux ${ }^{16}$, S. M. Rayner ${ }^{3}$, I. Redondo ${ }^{10,20, \star \star}$, A. Reimer ${ }^{17}$, O. Reimer ${ }^{17}$, J. Ripken ${ }^{5}$, L. Rob $^{18}$, L. Rolland ${ }^{16}$, G. Rowell ${ }^{1}$, V. Sahakian ${ }^{2}$, L. Saugé ${ }^{13}$, S. Schlenker ${ }^{7}$, R. Schlickeiser ${ }^{17}$, C. Schuster ${ }^{17}$, U. Schwanke ${ }^{7}$, M. Siewert ${ }^{17}$, H. Sol ${ }^{8}$, R. Steenkamp ${ }^{19}$, C. Stegmann ${ }^{7}$, J.-P. Tavernet ${ }^{16}$, R. Terrier ${ }^{6}$, C. G. Théoret ${ }^{6}$, M. Tluczykont ${ }^{10,20}$, G. Vasileiadis ${ }^{9}$, C. Venter ${ }^{15}$, P. Vincent ${ }^{16}$, H. J. Völk ${ }^{1}$, and S. J. Wagner ${ }^{12}$

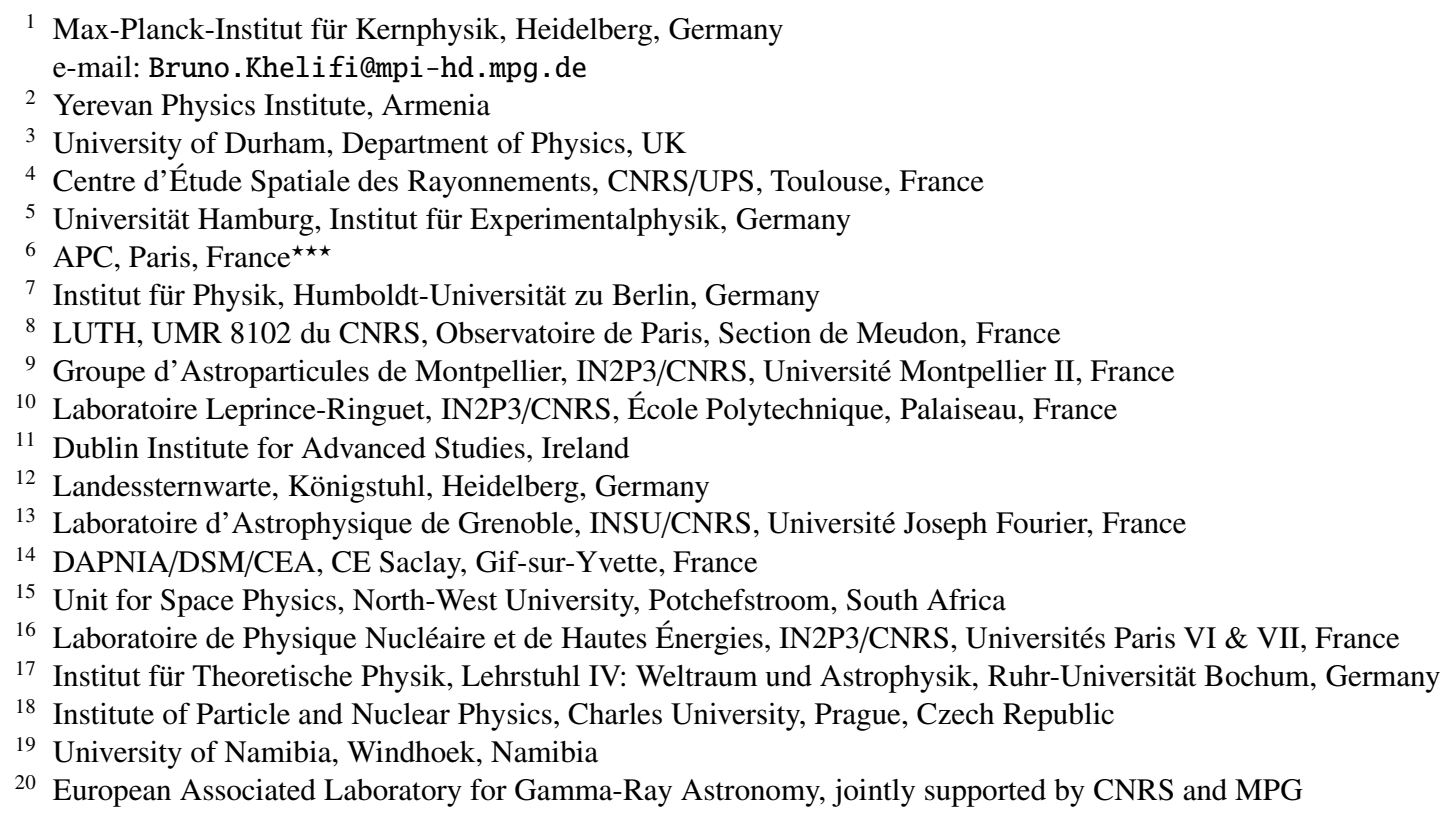

Received 2 March 2005 / Accepted 27 March 2005

\footnotetext{
* Université Libre de Bruxelles, Faculté des Sciences, Campus de la Plaine, CP230, Boulevard du Triomphe, 1050 Bruxelles, Belgium.

$\star \star$ Now at Department of Physics and Astronomy, Univ. of Sheffield, The Hicks Building, Hounsfield Road, Sheffield S3 7RH, UK.

$\star \star \star$ UMR 7164 (CNRS, Université Paris VII, CEA, Observatoire de Paris).
} 
Abstract. The Supernova Remnant MSH 15-52 has been observed in very high energy (VHE) $\gamma$-rays using the HESS 4-telescope array located in Namibia. A $\gamma$-ray signal is detected at the 25 sigma level during an exposure of $22.1 \mathrm{~h}$ live time. The image reveals an elliptically shaped emission region around the pulsar PSR B1509-58, with semi-major axis $\sim 6^{\prime}$ in the NW-SE direction and semi-minor axis $\sim 2^{\prime}$. This morphology coincides with the diffuse pulsar wind nebula as observed at X-ray energies by ROSAT. The overall energy spectrum from $280 \mathrm{GeV}$ up to $40 \mathrm{TeV}$ can be fitted by a power law with photon index $\Gamma=2.27 \pm 0.03$ stat $\pm 0.20_{\text {syst. }}$. The detected emission can be plausibly explained by inverse Compton scattering of accelerated relativistic electrons with soft photons.

Key words. gamma-rays: observations - ISM: supernova remnants - pulsar wind nebula - ISM: individual objects: PSR B1509-58 ISM: individual objects: G 320.4-1.2

\section{Introduction}

The Supernova Remnant (SNR) MSH 15-52 (G 320.4-1.2), first observed as an extended non-thermal radio source by Mills et al. (1961), is a complex object with an unusual morphology. Radio observations by Caswell et al. (1981) reveal a roughly circular $S N R \sim 30^{\prime}$ in diameter with a bright feature in the NW rim and a fainter one in the SE. The $10^{\prime}$ diameter NW source (G320.4-1.0) coincides with the $\mathrm{H}_{\alpha}$ nebula RCW 89.

Einstein X-ray observations of MSH $15-52$ by Seward \& Harnden (1982) led to the discovery of the 150-ms pulsar PSR B1509-58 located within the SNR shell, surrounded by a diffuse extended non-thermal component. The existence of a pulsar wind nebula (PWN) was later confirmed by ROSAT (Trussoni et al. 1996; Brazier \& Becker 1997) and ASCA (Tamura et al. 1996) as an extended emission region surrounding the pulsar with a power-law photon index of $\sim 2.0$. The PWN morphology is clearly visible in the ROSAT PSPC data (Trussoni et al. 1996) which show an elongated structure roughly centered on the pulsar with two arms extending several arcminutes along the NW and SE directions. These features were more recently confirmed by detailed Chandra observations (Gaensler et al. 2002).

Gaensler et al. (1999), comparing X-ray and radio observations, concluded that the radio SNR MSH 15-52 and the pulsar PSR B1509-58 are parts of a single system interacting via a pair of opposed collimated outflows (i.e. the PWN). The distance to the object and the pulsar spindown age were estimated to $5.2 \pm 1.4 \mathrm{kpc}$ and 1700 years, respectively.

The PWN hard X-ray spectrum was measured by BeppoSAX (Mineo et al. 2001) and is fitted by a power law with photon index of $2.08 \pm 0.01$ in the $1-200 \mathrm{keV}$ range. Interpreted as synchrotron emission from electrons within the PWN, these results suggest the presence of electrons accelerated up to energies of tens of $\mathrm{TeV}$, possibly leading to VHE $\gamma$-ray emission through an inverse Compton (IC) process as suggested by du Plessis et al. (1995). Early observations by the CANGAROO experiment (Sako et al. 2000) yielded a marginal $\gamma$-ray signal above $1.9 \mathrm{TeV}$ corresponding to $\sim 10 \%$ of the flux of the Crab Nebula.

\section{HESS observations and results}

MSH 15-52 was observed using the atmospheric Cherenkov technique with the full 4-telescope HESS (High Energy Stereoscopic System) array (Hinton 2004). The observations were made between March and June 2004 in wobble mode, for which the target position is offset by $0.5^{\circ}$ with respect to the

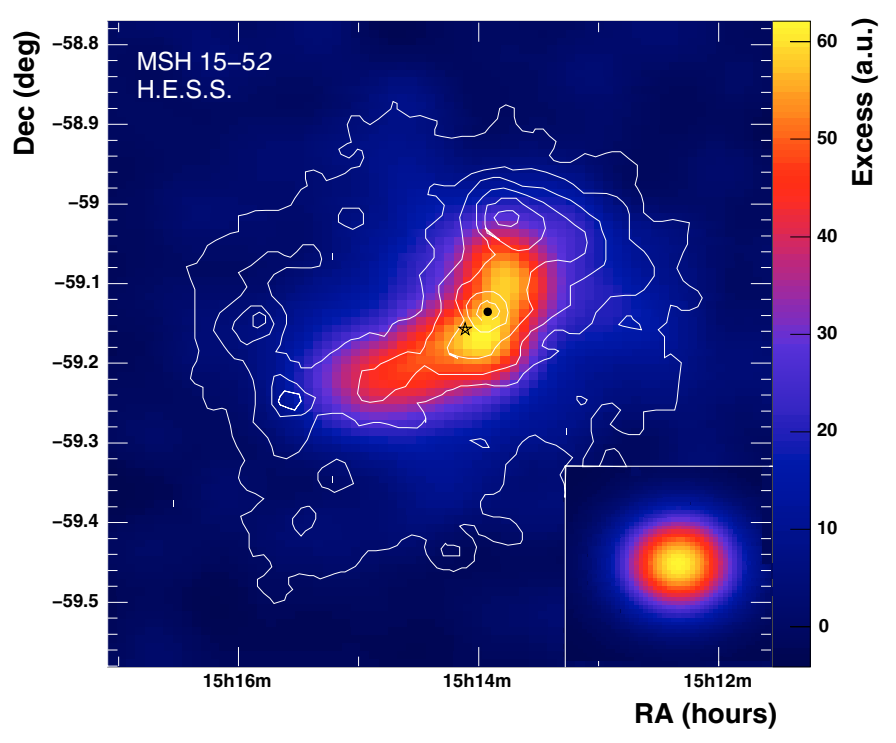

Fig. 1. Smoothed excess map from MSH 15-52 in arbitrary units (au). The map is smoothed with a Gaussian of $\sigma=0.04^{\circ}$ and only events with image sizes above 400 p.e. are used in order to improve the HESS angular resolution. The white contour lines denote the X-ray (0.6-2.1 keV) count rate measured by ROSAT (Trussoni et al. 1996). The black point and black star lie at the pulsar position and at the excess centroid, respectively. The right-bottom inset shows the simulated PSF smoothed identically.

centre of the field of view. Only data with good atmospheric conditions were selected yielding a total live time of $22.1 \mathrm{~h}$. The selected data were taken at a mean zenith angle of $37^{\circ}$. A standard analysis is used for the selection of $\gamma$-ray candidates, for the stereoscopic direction reconstruction of showers, and for spectral analysis (Aharonian et al. 2005), resulting in an average energy threshold of $\sim 280 \mathrm{GeV}$ at this zenith angle. For each sky bin $\left(0.6^{\prime} \times 0.6^{\prime}\right)$, the background level is estimated from events falling in a ring centered on this test position with a mean radius of $1^{\circ}$ and with an area 7 times that of the cell.

An excess with a significance of $\sim 25 \sigma$ (using the likelihood method of Li \& Ma 1983) is detected within the region of radius $0.14^{\circ}$ centered at the pulsar position with the standard point source analysis. Figure 1 shows the excess map of $\gamma$-ray candidates with image sizes greater than 400 photoelectrons (p.e.). The latter cut is meant to reduce the angular resolution to $\leq 0.07^{\circ}$ (70\% containment radius) and raises the energy threshold to $\sim 900 \mathrm{GeV}$. The map is convolved with a Gaussian of $\sigma=0.04^{\circ}$ in order to smooth out statistical fluctuations. An extended emission is seen along the jet axis of the pulsar (Trussoni et al. 1996) in the NW-SE direction. 
There is no evidence for VHE $\gamma$-ray emission from the shell of SNR G 320.4-1.2, including the NW part (RCW 89) from which a combination of thermal and non-thermal emission was seen in X-rays by ASCA (Tamura et al. 1996) and Chandra (Gaensler et al. 2002).

The unsmoothed excess map (with image sizes above 400 p.e.) is fitted by a two-dimensional Gaussian convolved with the point spread function (PSF) in order to extract the intrinsic dimensions. The best fit position of the Gaussian centroid is $\left(15 \mathrm{~h} 14 \mathrm{~m} 7 \mathrm{~s} \pm 21 \mathrm{~s},-59^{\circ} 9^{\prime} 27^{\prime \prime} \pm 11^{\prime \prime}\right)$ which is displaced by more than $3 \sigma$ from the pulsar position $(15 \mathrm{~h} 13 \mathrm{~m} 55.6 \mathrm{~s}$, $-59^{\circ} 8^{\prime} 8.9^{\prime \prime}$ ) taking into account the $20^{\prime \prime}$ systematic error on the pointing in each direction. The fitted direction of the major axis is $41^{\circ} \pm 13^{\circ}$ with respect to the RA axis, and is compatible with the X-ray direction $\left(60^{\circ} \pm 5^{\circ}\right)$ observed by Chandra. The intrinsic standard deviations along the major and minor axis are $6.4^{\prime} \pm 0.7^{\prime}$ and $2.3^{\prime} \pm 0.5^{\prime}$, respectively. These dimensions are comparable to those of the diffuse PWN as observed by ROSAT, $\sim 10^{\prime} \times 6^{\prime}$ (Trussoni et al. 1996). Figure 2 shows the projection along the major and minor axis of the count map relative to the pulsar position and illustrates the morphological features described above. The transverse profile is approximately symmetric. The longitudinal profile, in contrast, appears to extend more in the SE than in the NW direction relative to the pulsar position. Given the spatial coincidence and the similar morphology of the HESS picture and the X-ray data, we identify this new VHE $\gamma$-ray source with the pulsar wind nebula in MSH 15-52.

The total $\gamma$-ray excess within a circle centered on the Gaussian centroid with a radius of $0.3^{\circ}$, which encompasses the entire VHE $\gamma$-ray emission, is $3481 \pm 129$ events after standard cuts. Figure 3 shows the reconstructed spectrum from these events. The data are consistent with a power law $\left(\mathrm{d} N / \mathrm{d} E \propto E^{-\Gamma}\right)$ up to $\sim 40 \mathrm{TeV}$ with a photon index of $\Gamma=$ $2.27 \pm 0.03_{\text {stat }} \pm 0.20_{\text {syst }}$ and a differential flux at $1 \mathrm{TeV}$ of $\left(5.7 \pm 0.2_{\text {stat }} \pm 1.4_{\text {syst }}\right) \times 10^{-12} \mathrm{TeV}^{-1} \mathrm{~cm}^{-2} \mathrm{~s}^{-1}$. The $\chi^{2} /$ d.o.f. of the fit is 13.3/12. The corresponding integral flux above $280 \mathrm{GeV}$ represents $\sim 15 \%$ of the Crab Nebula flux above the same threshold. The flux distribution, as measured on a run by run basis, is compatible with steady emission (the $\chi^{2} /$ d.o.f. is $47.0 / 51$ ).

\section{Discussion and conclusions}

The HESS observations of MSH 15-52 provide the first image of an extended PWN in the VHE range. The emission region is clearly extended along the jet axis. If this VHE $\gamma$-ray emission arises from inverse Compton (IC) scattering of target photons by electrons, the latter should emit synchrotron radiation below $1 \mathrm{keV}$. Thus, the morphology similar to that seen in soft X-rays by ROSAT suggests a leptonic origin of the HESS signal. Assuming the target photons are uniformly distributed, IC emission directly reflects the distribution of high-energy electrons, unlike synchrotron emission which also reflects spatial variations of the magnetic field.

The lack of VHE $\gamma$-ray emission in the NE-SW direction shows that the elongated morphology of the PWN in X-rays is not due to a lower magnetic field strength, $B$, further away
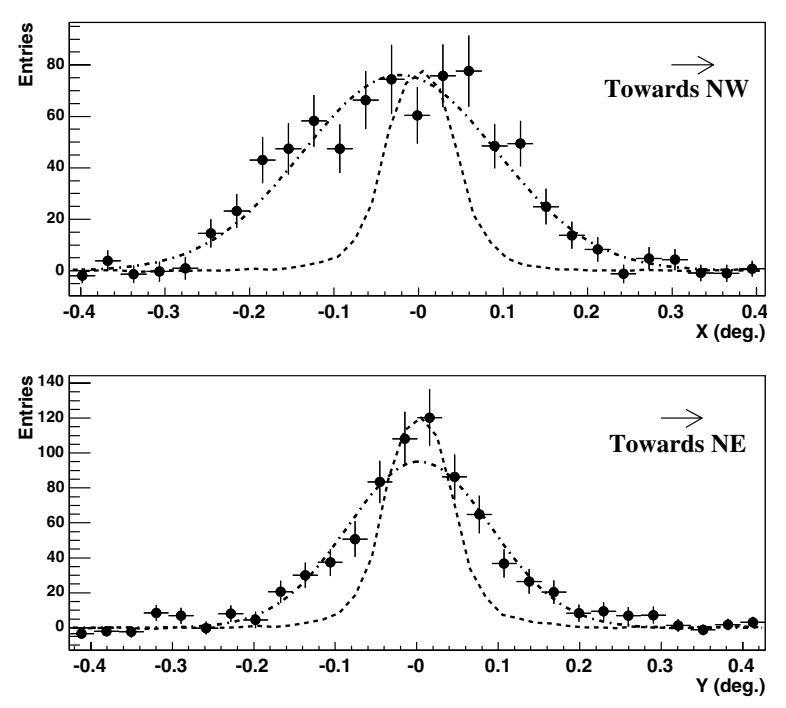

Fig. 2. Projection of the unsmoothed excess map along the major (top) and minor (bottom) axes relative to the pulsar position. The dot-dashed lines are the best-fit Gaussians to these distributions, and the dashed lines show the distribution for a Monte-Carlo point source excess at the pulsar position.

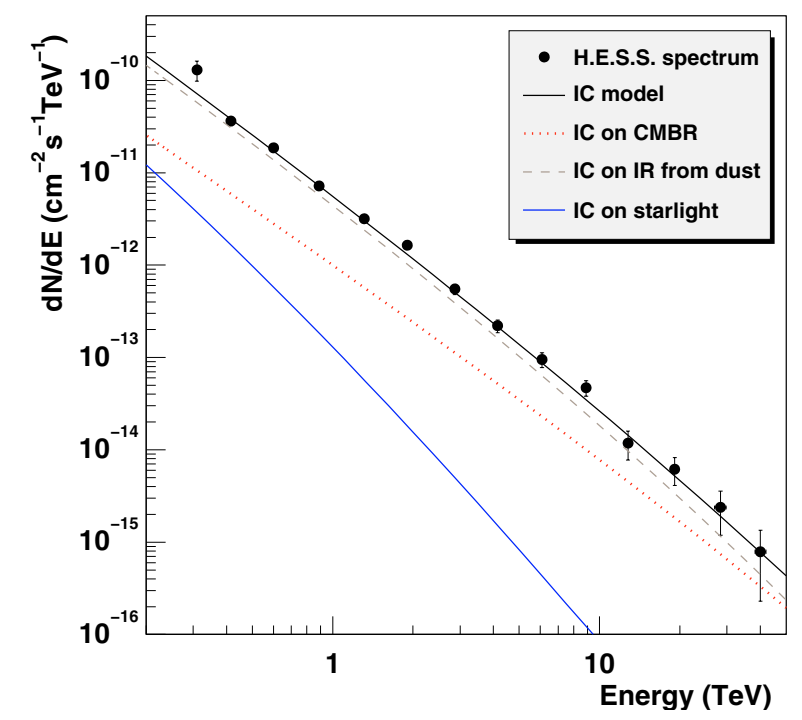

Fig. 3. Reconstructed VHE $\gamma$-ray spectrum of the whole nebula. The lines are the best model fit of the one zone model described in Sect. 3.

from the jet axis. However the reason for the apparent lack of high-energy electrons in the equatorial direction is not clear. If the spin axis is aligned with the magnetic axis of the pulsar, as suggested by Brazier \& Becker (1997), Sulkanen \& Lovelace (1990) show that most of the energy, angular momentum and electric current are carried within the jet, which may enhance the accelerated electron density within jets. The VHE $\gamma$-ray emission asymmetry along the jet axis relative to the pulsar may be interpreted as Doppler boosting within the two opposite jets (Pelling et al. 1987). The two jets may also be confined differently by the surrouding medium, as suggested by the appearance of RCW 89 to the NW.

The total power, $L$, radiated by MSH $15-52$ in the energy band $0.3-40 \mathrm{TeV}$ is $\sim 1.0 \times 10^{35} \mathrm{erg} \mathrm{s}^{-1}$ (here we adopt a distance of $5 \mathrm{kpc}$ ) compared to $\sim 8.0 \times 10^{34} \mathrm{erg} \mathrm{s}^{-1}$ for the 
Crab Nebula (assuming a distance of $2 \mathrm{kpc}$ ). Thus, the efficiency of VHE $\gamma$-ray production relative to the spin-down rate $(L / \dot{E})$ of PSR B1509-58 $(\sim 0.6 \%)$ is much larger than that of the Crab pulsar $(\sim 0.016 \%)$. Such high efficiency has been proposed previously (Aharonian et al. 1997).

A simple one-zone IC model (Khélifi 2002) can be used to reproduce the VHE spectrum of the whole nebula. Therein, a population of accelerated electrons with a power-law energy spectrum is assumed. These particles lose their energy by synchroton emission in a magnetic field, assumed to be uniform within the PWN, and by IC scattering on seed photons. The electron spectrum and the magnetic field strength are adjusted such that the computed synchrotron and IC spectra match the X-ray and VHE $\gamma$-ray observations. The energy spectrum of the whole nebula measured by BeppoSAX (Mineo et al. 2001) is used for this fit. By using only the cosmic microwave background radiation (CMBR) as seed photons, it is not possible to reproduce the VHE spectrum. Thus, infrared (IR) photons from dust and starlight are added, using a recent parametrisation of the interstellar radiation field (ISRF) used for the GALPROP code (Strong et al. 2000). The energy density of the dust and starlight components of the ISRF are however kept free to account for possible local variations.

The best fit model is represented in Fig. 3 in the VHE band with the contribution of the different seed photons to the IC spectrum. The fitted spectral index of radiating electrons is 2.9. The fitted mean magnetic field strength is $\sim 17 \mu \mathrm{G}$, which is about a factor of two higher than the lower limit estimated previously (Gaensler et al. 2002). The best fit energy density of the dust component is about $2.3 \mathrm{eV} \mathrm{cm}^{-3}$ which is more than twice the nominal value of the GALPROP ISRF. This apparent enhancement of dust density may be due to the material swept up by the SNR G 320.4-1.2 (du Plessis et al. 1995). As the energy density of the starlight component is unconstrained, it is fixed to the GALPROP value $\left(\sim 1.4 \mathrm{eV} \mathrm{cm}^{-3}\right)$.

Although this spectral model is compatible with all available measurements, the derived value of $B$, when combined with the pulsar spin-down age of 1700 years, suggests the existence of a cooling break in the electron spectrum of $\sim 24 \mathrm{TeV}$. This corresponds to a photon energy above $\sim 3 \mathrm{TeV}$ in the IC spectrum, depending on the energy of the seed photons, which should be detectable. Nonetheless, if the age of the system is closer to 20000 years as suggested by Gvaramadze (2001), the above spectral model would be self-consistent. It should also be noted that there is noticeable correlation between the fitted $B$ value and the photon energy density from dust. Thus, if this local density was higher, the corresponding $B$ value would increase and thus the cooling break energy in the IC spectrum would decrease.

In summary, this detection represents the first morphological identification of an extended pulsar wind nebula in the VHE $\gamma$-ray band. The HESS observations provide more direct information on accelerated particles than soft X-ray data, due to the uncertainties in the estimation of the magnetic field and its spatial variations.

Acknowledgements. The support of the Namibian authorities and of the University of Namibia in facilitating the construction and operation of HESS is gratefully acknowledged, as is the support by the German Ministry for Education and Research (BMBF), the MaxPlanck-Society, the French Ministry for Research, the CNRS-IN2P3 and the Astroparticle Interdisciplinary Programme of the CNRS, the UK Particle Physics and Astronomy Research Council (PPARC), the IPNP of the Charles University, the South African Department of Science and Technology and National Research Foundation, and by the University of Namibia. We appreciate the excellent work of the technical support staff in Berlin, Durham, Hamburg, Heidelberg, Palaiseau, Paris, Saclay, and in Namibia in the construction and operation of the equipment. We would also like to thank T. Mineo for provision of the BeppoSAX data in numerical form.

\section{References}

Aharonian, F., Akhperjanian, A. G., Aye, K.-M., et al. 2005, A\&A, 430,865

Aharonian, F., Atoyan, A. M., \& Kifune, T. 1997, MNRAS, 291, 162

Brazier, K. T. S., \& Becker, W. 1997, MNRAS, 284, 335

Caswell, J. L., Milne, D. K., \& Wellington, K. J. 1981, MNRAS, 195, 89

du Plessis, I., de Jager, O. C., Buchner, S., et al. 1995, ApJ, 453, 746

Gaensler, B. M., Brazier, K. T. S., Manchester, R. N., Johnston, S., \& Green, A. J. 1999, MNRAS, 305, 724

Gaensler, B. M., Arons, J., Kaspi, V. M., et al. 2002, ApJ, 569, 878

Gvaramadze, V. V. 2001, A\&A, 374, 259

Hinton, J. A. (for the HESS Collaboration) 2004, New Astron. Rev., 48,331

Khélifi, B. 2002, Ph.D. Thesis, University of Caen, France http://lpnp90.in2p3.fr/ cat/Thesis/

Li, T., \& Ma, Y. 1983, ApJ, 272, 317

Mills, B. Y., Slee, O. B., \& Hill, E. R. 1961, Austr. J. Phys., 14, 497

Mineo, T., Cusumano, G., Maccarone, M. C., et al. 2001, A\&A, 380, 695

Pelling, R. M., Paciesas, W. S., Peterson, L. E., et al. 1987, ApJ, 319, 416

Rodgers, A., Campbell, C. T., \& Whiteoak, J. B. 1960, MNRAS, 121, 103

Sako, T., Matsubara, Y., Muraki, Y., et al. 2000, ApJ, 537, 422

Seward, F. D., \& Harnden, F. R., Jr. 1982, ApJ, 256, L45

Strong, A. W., Moskalenko, I. V., \& Reimer, O. 2000, ApJ, 537, 763 S

Sulkanen, M. E., \& Lovelace, R. V. E. 1990, ApJ, 350, 732

Tamura, K., Kawai, N., \& Yoshida, A. 1996, Publ. Astron. Soc. Jpn, 48, L33

Trussoni, E., Massaglia, S., Caucino, S., Brinkmann, W., \& Aschenbach, B. 1996, A\&A, 306, 581 